\title{
The role of health care workers and antiviral drugs in the control of pandemic influenza is
}

\author{
Belinda Barnes *, Kathryn Glass, Niels G. Becker \\ National Centre for Epidemiology and Population Health, Australian National University, Canberra 0200, Australia
}

Received 30 November 2006; received in revised form 14 February 2007; accepted 19 February 2007

Available online 2 March 2007

\begin{abstract}
Until a vaccine against the new strain becomes available, the response to newly emerged pandemic influenza will consist of the use of antiviral drugs and measures that limit exposure to infectious individuals. These first-line defence measures include isolating cases upon diagnosis, reducing close contacts, the use of personal protective equipment and hygiene, and using antiviral drugs for treatment and prophylaxis. There are significant 'costs' associated with control measures, so to justify such interventions it is important to assess their potential to reduce transmission. In this paper, we determine the effect that a number of different antiviral interventions have on the reproduction number of infectives and the probability that an imported infection fades out, and determine parameter scenarios for which these interventions are able to eliminate an emerging pandemic of influenza. We also assess the role that health care workers play in transmission and the extent to which providing them with antiviral prophylaxis and personal protective equipment modifies this role. Our results indicate that this class requires protection to avoid a greatly disproportionate contribution to early infective numbers, and for the maintenance of a stable health care system. Further, we show that the role children play in increasing transmission is moderate, in spite of closer mixing with other children.

(c) 2007 Elsevier Inc. All rights reserved.
\end{abstract}

\footnotetext{
Funding from Australian NHMRC Grants 358425 and 410224, and the Australian Department of Health and Ageing, is gratefully acknowledged.

Corresponding author. Tel.: +61 2 61258088; fax: +61 261250704.

E-mail address: Belinda.Barnes@anu.edu.au (B. Barnes).
} 
Keywords: Antiviral drugs; Basic reproduction number; Control of infectious disease outbreaks; Effective reproduction number; Elimination of an infection; Emerging infections; Epidemic control; Pandemic influenza

\section{Introduction}

There is general acceptance that another substantial shift in the influenza virus will occur, leading to a pandemic. The current global spread of the avian influenza virus H5N1 and its transmission from birds to humans may make it happen sooner, rather than later. Accordingly, there is an urgency to be prepared for an influenza pandemic, although our degree of vulnerability and the disease characteristics are largely unknown [1]. A vaccine will take time to develop, and due to high demand may be in short supply globally [2]. Thus all available first-line defence strategies should be carefully assessed for effectiveness and feasibility.

Initial defence strategies to contain transmission include minimizing contacts with infected and potentially infected individuals, and careful use of the limited stockpile of antiviral drugs. Early detection of infected individuals with subsequent isolation and quarantining individuals exposed to them, as well as the closure of schools, are well known strategies for reducing transmission. However, decisions concerning such interventions can be controversial because of the cost and inconvenience they impose, particularly when there are doubts as to their effectiveness. In this paper, we assess how effectively various control measures involving the use of antiviral drugs are able to contribute to eliminating a newly emerged influenza infection.

Becker et al. [3] assess the effectiveness of interventions to reduce the reproduction number of a newly emerged infectious disease with characteristics like SARS. Here we conduct a similar analysis with a focus on pandemic influenza, but with a number of important differences. First, we use disease characteristics of influenza, which differ from those of SARS. Second, we consider the impact of protecting health care workers (who mix closely with infectives) against infection. Third, we explore the effectiveness of using antiviral drugs to reduce transmission and the probability that an importation leads to a major outbreak. The effect of antiviral drugs is complex because they can be used both for treatment and for prophylaxis, and stockpiles of such drugs are limited. Furthermore, antiviral drugs might be dispensed for prophylaxis prior to exposure to the infection, or afterwards. Examples of these are, respectively, antivirals given to health care workers for their protection and antivirals given to members of a household upon diagnosis of a primary case within the household. We also explore the role that children (who mix more closely with their peers than with adults) play in transmitting infection.

Longini et al. [4], Ferguson et al. [5], Wu et al. [6] and Germann et al. [7], have considered the impact on disease transmission of antiviral drug administration. Their motivation, as here, was to establish conditions under which the disease is contained. These papers approach the modelling question through micro-simulation based on individual characteristics and mixing behaviour; the first for the Hong Kong population of 6.8 million, the next two for populations of 281 million and 2000, respectively, in the United States, and the last for a population of 85 million individuals in Thailand. The advantage of their approach, as well as the dynamical systems approach of [8] for populations in the US, UK and the Netherlands, is that it enables prediction of case numbers, 
antiviral stockpile depletion and other dynamics in calendar time, while also providing forecasts of epidemic size at any time and the period of time over which the epidemic runs its course.

In contrast, we focus on two concepts that are central to the control of an emerging infectious disease, namely the effective reproduction number $(R)$ and the probability that an importation fails to initiate a local epidemic $(q)$. The advantages of limiting attention to $R$ and $q$ are that we require fewer assumptions, some analytic results are possible and calculations are far less computer intensive. As a consequence, a comprehensive check of how sensitive results are to assumptions about parameter values and model structure becomes feasible. However, our approach does not capture the calendar-time dynamics of the epidemic, so micro-simulation and the approach used here complement each other.

The reproduction number $R$ is central to the control of an infectious disease because it measures the rate at which infectives are able to multiply and, importantly, $R<1$ indicates that local transmission of the infection will fade out with certainty. Our aim is to determine the extent to which the reproduction number is reduced by various control measures involving antiviral drug distribution and, specifically, to determine intervention requirements to bring its value below unity. In the case that $R$ is reduced, but remains above unity, we consider the impact of the strategy on the probability of disease elimination, $q$.

We base our assessment on transmission models that, to accommodate the above-mentioned interventions, distinguish between several types of individual, namely children, adults and certain health care workers. We formulate our model in Section 2. In Section 3 we consider the impact of targeting the contacts of every diagnosed infective with antiviral drugs, where such drug administration is either pre- or post-exposure. Subsequently, in Section 4, we examine the role of influenza-dedicated health care workers on disease transmission and early case numbers. We look at what effect providing antiviral drugs to this group, as well as their use of personal protection equipment (PPE), has on disease transmission. In Section 5 we include children and determine the impact of their increased mixing. Providing them with antiviral drugs as prophylaxis or vaccinating them as a group are the interventions we consider. Finally, we conclude with some general observations (Section 6).

\section{Model and reproduction number}

We assume that infection is spread primarily by person-to-person transmission. To begin with let us assume that members of the general public are homogeneous, in that each is equally likely to become infected. The infection is assumed to be accompanied by an illness that leads each case to present to a general practitioner, who may be infected during this consultation. Immediately following presentation the infective is isolated in a hospital and cared for by influenza-dedicated health care workers. With these assumptions we require three distinct types of individual; members of the general public (Type $G$ ), general practitioners (Type $D$ ) and influenza-dedicated health care workers (Type $H$ ).

Let $m$ denote the mean number of community members infected by an infective during the infectious period, when every contacted person is susceptible and no interventions are in place. With isolation the mean number of community members infected becomes $m f$, where $f$ is the fraction of the infection potential spent before being isolated and we assume diagnosis occurs immediately with consultation. When the infectiousness of an infective is constant over the 
infectious period, $f$ is simply the fraction of the infectious period experienced before isolation. The probability that the general practitioner is infected when an infective presents is $p$. An infected general practitioner also infects a mean of $m f$ individuals while mixing with community members. The remaining infection potential, namely $(1-f) m$, acts on Type $H$ individuals who are caring for the isolated infectives. The mixing with carers differs from mixing with community members, as carers take precautions in their contacts with patients. Hence the mean number of Type $H$ individuals an infective infects is assumed to be $(1-f) m e_{p}$, where $e_{p}$ adjusts for the changed mixing and the precautions Type $H$ individuals take during contacts, including protection provided by wearing personal protective equipment (PPE). It is assumed that general practitioners and influenza-dedicated health care workers do not infect a general practitioner at presentation, because they either self-diagnose or present in a way that minimises the chance of transmission. No increased rate of transmission between health care workers is included in the model.

Antiviral drugs have been shown to reduce susceptibility and infectivity for seasonal influenza, when used for prophylaxis. We assume that they will similarly reduce transmission of the emerged pandemic strain of influenza. Let $e_{s}$ denote the factor by which the per-contact probability of infection is reduced for a susceptible individual who is using antivirals for prophylaxis, relative to an individual who is not. Antivirals offer no protection if $e_{s}=1$ and are fully protective if $e_{s}=0$. Also, let $e_{i}$ be the factor by which the infection potential is reduced for an individual who is using antivirals for prophylaxis and becomes infected, relative to an infective not on antiviral drugs. Antivirals provide no reduction in infectivity if $e_{i}=1$ and reduce infectivity to zero if $e_{i}=0$. We adopt as default intervention (referred to below as 'default AVs'), one in which doctors and influenza-dedicated health care workers are provided with antiviral drugs as prophylaxis, health care workers are also provided with PPE and diagnosed patients are given antiviral drugs as treatment. This level of intervention seems essential to maintain health care services. Treated patients are assumed to have their remaining infection potential reduced by a factor $e_{i}$.

We seek to determine disease characteristics for which different levels of intervention are able to eliminate the infection. Therefore, we can, without loss of generality, look at transmission in terms of generations of infectives. The initial infective(s) make up generation zero. The individuals they infect make up generation one, and so on. The effective reproduction number is the largest eigenvalue of the next-generation mean matrix $M$ [9]. The word effective in 'effective reproduction number' refers to the fact that there is some intervention in place. This matrix allows computation of the mean number of infectives in one generation from the number of cases in the previous generation.

To define $M$, and examine strategies involving a proportion $g$ of Type $G$, we divide this group into two subgroups, $G_{1}$ and $G_{2}$, and introduce $\alpha$, a mixing parameter, to allow distinct mixing patterns between these subgroups. A general form of $M$ that incorporates a number of health care strategies is

$$
M=\left(\begin{array}{cccc}
f m(1-g) & f m \tilde{e}_{i}(1-g) & f m e_{i}(1-g) & f m e_{i}(1-g) \\
f m \tilde{e}_{s} g & f m \alpha \tilde{e}_{i} \tilde{e}_{s} g & f m e_{i} \tilde{e}_{s} g & f m e_{i} \tilde{e}_{s} g \\
p & 1-(1-p)^{\tilde{e}_{i}} & 0 & 0 \\
(1-f) m e_{i} e_{s} e_{p} & (1-f) m e_{i} e_{s} e_{p} & (1-f) m e_{i} e_{s} e_{p} & (1-f) m e_{i} e_{s} e_{p}
\end{array}\right) .
$$

To illustrate its role as a next generation matrix note that if $\mathbf{x}_{\mathbf{0}}=\left(G_{10} G_{20} D_{0} H_{0}\right)$ gives the number of Type $G_{1}$, Type $G_{2}$, Type $D$ and Type $H$ infectives in generation zero, then the mean number of 
infectives of types $G_{1}, G_{2}, D$ and $H$ in generation one is given by $M \mathbf{x}_{\mathbf{0}}{ }^{T}$ (where $T$ denotes the transpose). Below, parameters $\tilde{e}_{i}$ and $\tilde{e}_{s}$ will be either unity or $e_{i}$ and $e_{s}$, respectively, depending on the intervention implemented.

The effective reproduction number $R$, given by the leading eigenvalue of $M$, is (see Appendix A)

$$
R \approx f m(1-g)+f m \alpha \tilde{e}_{i} \tilde{e}_{s} g+(1-f) m e_{i} e_{s} e_{p}=\operatorname{Trace}(M)
$$

The basic reproduction number $R_{0}$, which is the reproduction number when there is no intervention in place, is obtained by substituting $f=e_{i}=e_{s}=e_{p}=\tilde{e}_{i}=\tilde{e}_{s}=1$.

Although $R$ does not impart all information concerning the dynamics in calendar time, it does provide a valuable means by which to compare the impact of different intervention strategies; the manner in which it is applied in this paper.

\subsection{Parameter values and sensitivity analysis}

Table 1 provides a glossary of all parameters used in this paper, some introduced above and others below in the relevant sections, together with their default values.

Estimates of $R_{0}$ for pandemic influenza given in the literature [10] are mostly low compared with estimates of $R_{0}$ for other infectious diseases. They range from close to 2, as applied in $[4,5]$, to values between 2 and $3[11,10]$. Here we do not fix $R_{0}$, but rather consider a wide interval that encompasses these estimates.

Proportions of the public in different classes are estimated from Australian data. The proportion of children is calculated from 2001 census data, and the proportion of doctors and health care

Table 1

Model parameters with default values

\begin{tabular}{llc}
\hline Param. & Description & Default value(s) \\
\hline$m$ & Basic reproduction number with neither intervention \\
$R_{0}$ & $\begin{array}{l}\text { nor medical consultations in a fully susceptible population } \\
\text { Basic reproduction number without intervention, } \\
\text { but with medical consultations in a fully susceptible population }\end{array}$ \\
$q$ & Effective reproduction number with intervention \\
$q$ & Probability that an outbreak with 1 initial case is minor & \\
$p$ & Fraction of an individual's infectivity spent mixing & 0.5 \\
$e_{i}$ & Probability of infection at consultation & 0.008 \\
$e_{S}$ & Reduction in infectivity when taking AVs & 0.3 \\
$e_{p}$ & Reduction in susceptibility when taking AVs & 0.25 \\
$g$ & Reduction in susceptibility when using PPE & 0.25 \\
$\alpha$ & Proportion of general public involved in an intervention & $0,0.4,0.8$ \\
$\pi_{G}$ & Factor by which mixing differs in group $G_{2}$ & $1,1.9,5.4$ \\
$\pi_{D}$ & Proportion of public who are not HCWs & 0.988 \\
$\pi_{H}$ & Proportion of public who are doctors & 0.002 \\
$c$ & Proportion of public who are influenza-dedicated HCWs & 0.01 \\
\hline
\end{tabular}


workers from a medical labour force survey. We assume that around $20-25 \%$ of the available health care workers are at high risk of infection and may be given antivirals as prophylaxis. These individuals are referred to as influenza-dedicated health care workers.

Parameters describing the effectiveness of antivirals are based on trials of Oseltamivir. We assume a default value for the reduction in infectiousness while on antivirals of 0.3 , which is consistent with studies of antiviral efficacy and other modelling work [5,12-14]. Although some studies suggest that antivirals are not particularly effective against the broad category of influenza-like illness [14], trials assessing effectiveness against influenza have shown relatively high reductions in susceptibility $[13,15,16]$. As we are considering interventions adopted during an influenza pandemic, we use the latter data to set a default value for the reduction in susceptibility while on antivirals of 0.25 . Influenza-dedicated health care workers may be able to reduce their probability of infection further by using personal protective equipment (PPE) such as masks, gloves, hygiene practices and changed mixing patterns. We assume that the reduction in susceptibility of an individual using PPE is 0.25 [17,18]. We note that our results are highly sensitive to the values of these parameters, thus highlighting the importance of accurate estimates of efficacy in the event of a pandemic.

For $p$, the probability of infection at a consultation, we assume a default value of 0.008 . We found that over the range $0.005-0.02$ (which includes attack rates in doctors of close to $100 \%$ ), there was little effect on the results presented here.

Our models incorporate some additional transmission between children to take account of differences in infectiousness, susceptibility or mixing patterns that may exist. The parameter governing this extra mixing $(\alpha)$ was chosen so that age-specific attack rates matched those observed in the pandemics of 1957 and 1968/9 [19], giving two potential values: $\alpha=1.9$ corresponding to the $1968 / 9$ data, and $\alpha=5.4$ corresponding to the 1957 data. In our analysis we consider both these values of $\alpha$, as well as $\alpha=1$, thus quantifying the sensitivity of the model to this parameter.

Parameter $f$ denotes the proportion of an individual's infectiousness that is exerted on the community before the infective is isolated. It is possible that the degree of infectiousness is constant over the infectious period, or that it varies, rising high early in the infectious period and then decreasing towards zero. These two infectivity profiles are referred to as 'flat' and 'peaked', respectively. Our peaked infectivity profile is similar to that used in [5]. For both profiles, $f$ denotes the proportion of the area below the infectiousness function curve before isolation. If a case presents and is isolated at the onset of symptoms, the value of $f$ corresponding to the flat profile will be significantly less than that for the peaked profile.

Our results were found to be quantitatively sensitive to $f$ and thus, for certain results, we consider the full range of $f \in[0,1]$. In other cases, $f=0.5$ has been chosen as an illustrative example, since for influenza it would be expected that $f \geqslant 0.5$. This is a consequence of the following. After infection, the latent period is approximately 1 day followed by an infectious period with symptoms appearing at, approximately, the beginning of the third day [20,5]. Infection lasts for approximately 5-6 days [20] and isolation would not be expected before day 4, implying that it is likely that at least half of the infectivity would have passed before isolation. This argument is strengthened by the fact that viral load is expected to be greater during the early stages of the infectious period [5]. For variation in this parameter $f$, comment is provided where relevant as to quantitative and qualitative differences in results. 


\section{Impact of providing antivirals to targeted contacts}

We address questions concerning antiviral drug interventions, and the impact of delayed distribution, through a relative assessment of three strategies, all of which include the 'default AVs' strategy:

- Strategy A: Suppose that a fraction $g$ of general community contacts are given drugs pre-exposure. For example, when a case arises in a location a proportion $g$ of individuals in that location are provided with antiviral drugs as prophylaxis, whence $\tilde{e}_{i}=e_{i}$ and $\tilde{e}_{s}=e_{s}$. Such an approach might be used early in an outbreak, when antiviral drugs are plentiful.

- Strategy B: Suppose antiviral drugs are distributed as above, beginning after the first generation. This means that no susceptibles are protected during the initial generation. Our motivation is that $q$ is largely determined by what occurs early in an outbreak, and this strategy indicates the substantial impact of even a very short delay in implementing Strategy A.

- Strategy C: Suppose antiviral drugs are distributed to a proportion $g$ of general community contacts post-exposure, on presentation of the infective. The strategy indicates the impact of a more targeted use of antivirals and is foreseeable when stockpiles of antiviral drugs are limited. Due to the nature of influenza this strategy can be approximated with contacts not being protected when susceptible $\left(\tilde{e}_{s}=1\right)$, but their subsequent infectivity being reduced $\left(\tilde{e}_{i}=e_{i}\right)$. In contrast to Strategy A, Strategy C administers antiviral drugs to contacts post-exposure.

Note that antiviral drugs are assumed to be distributed in a timely manner so that their full efficacy is realised.

Fig. 1 compares the impact of Strategies $\mathrm{A}$ and $\mathrm{C}$ on transmission, as reflected by $R$. Our results suggest that $g \geqslant 0.6$ under Strategy $A$, and $g \geqslant 0.8$ under Strategy $C$, have a considerable impact

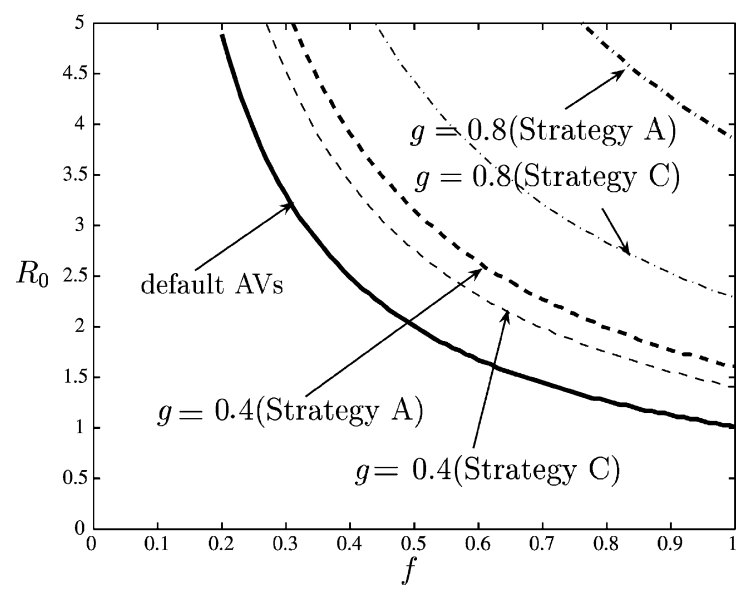

Fig. 1. The impact of using antivirals for prophylaxis expressed by contours for $R=1$ : above the curves there is a chance that the disease takes off, while below, the outbreak is minor with certainty. All strategies include the 'default AVs' intervention. Solid curve: the 'default AVs' intervention alone; dashed curves: antiviral Strategy A (pre-exposure distribution of AVs); dash-dotted curves: antiviral Strategy C (post-exposure distribution of AVs). (Parameters as in Table 1 with $\alpha=1$.) 
on reducing transmission, and such levels of contact identification are thought to be achievable $[4,21]$. Note that for larger values of $f$, as might be associated with the peaked infectiousness function, the effect is less pronounced. These results indicate that providing antiviral drugs to contacts is an effective and feasible means of reducing transmission, and that, for both strategies considered, as $g$ increases so the reduction in transmission increases exponentially. While there is an appreciable benefit to providing the drugs prior to exposure, providing antivirals for prophylaxis could be wasteful of a limited stockpile.

Our results on the effectiveness of antiviral drugs are in general agreement with those of the dynamical systems model proposed in [8]. Although the modelling approaches are quite different, the effective reproduction numbers associated with the distribution to a proportion of the general population are very similar.

The probability $(q)$ of no influenza outbreak when $R>1$ may be greater than zero, and thus, to complement Fig. 1, it is useful to quantify $q$. To do so we need to assume a form for the probability distribution of the number of individuals in each class that are infected by a single infective. Below we compare the results for two such distributions.

Assume that an infective initiates a multi-type branching process, infecting susceptibles in $G_{1}$, $G_{2}$ and $H$ according to a Poisson process, while offspring in $D$ are Bernoulli trials. The number of each type infected by an individual are independent with mean values given by the elements of $M$. Let $q_{i}$ (for $i=G_{1}, G_{2}, D, H$ ) be the conditional probability that the disease does not take off given an initial infective in population group $i$. Then, using standard methods based on probability generating functions,

$$
\begin{aligned}
& q_{G_{1}}=\mathrm{e}^{m f(1-g)\left(q_{G_{1}}-1\right)} \mathrm{e}^{m f \tilde{e}_{s} g\left(q_{G_{2}}-1\right)}\left[1+p\left(q_{D}-1\right)\right] \mathrm{e}^{m(1-f) e_{i} e_{s} e_{p}\left(q_{H}-1\right)} \\
& q_{G_{2}}=\mathrm{e}^{m f \tilde{e}_{i}(1-g)\left(q_{G_{1}}-1\right)} \mathrm{e}^{m f \tilde{e}_{i} \tilde{e}_{s} g\left(q_{G_{2}}-1\right)}\left[1+\left[1-(1-p)^{\tilde{e}_{i}}\right]\left(q_{D}-1\right)\right] \mathrm{e}^{m(1-f) e_{i} e_{s} e_{p}\left(q_{H}-1\right)} \\
& q_{D}=\mathrm{e}^{m f e_{i}(1-g)\left(q_{G_{1}}-1\right)} \mathrm{e}^{m f e_{i} \tilde{e}_{s} g\left(q_{G_{2}}-1\right)} \mathrm{e}^{m(1-f) e_{i} e_{s} e_{p}\left(q_{H}-1\right)}
\end{aligned}
$$

and $q_{H}=q_{D}$. With this formulation, and numerical methods, we consider the impact on $q_{G_{1}}$ of Strategies A, B and C, when compared with the strategy of 'default AVs' (Fig. 2(a)).

As expected, when compared with Strategy A, Strategy B does not change $R$; however, $q_{G_{1}}$ is reduced considerably. Strategy $\mathrm{C}$ (when compared with Strategy A) increases $R$ (Fig. 1) and reduces $q_{G_{1}}$. (For larger $f$, the results are qualitatively similar, although $q_{G_{1}}$ is reduced.) What is notable is that, even though $R>1$, the probability of a minor outbreak may remain high.

These results suggest that, for values of $R_{0}<3[11,4,5,10]$, the use of antiviral drugs to target a proportion of all contacts of infected cases is an effective means of reducing transmission and decreasing the chance that a single importation leads to a major outbreak, in spite of $R$ remaining greater than unity and the post-exposure distribution of antiviral drugs. From data on attack rates collected from previous influenza pandemics, $R_{0}$ is likely to be less than $2.5[11,4,5,7,10]$ and then the probability of avoiding a major epidemic is close to unity when $40 \%$ or more of all contacts are targeted, even for delayed intervention. If we accept it is feasible to identify, and provide antiviral drugs to, $80 \%$ of contacts [4,21], our results suggest than an epidemic can be avoided for an $R_{0}$ as high as 3 .

As an alternative consider a Negative Binomial offspring distribution, for which secondary cases occur through a Poisson process with the mean of this process Gamma distributed. It represents a broader class of distributions of which the Poisson is a limiting case. Application of this 
a

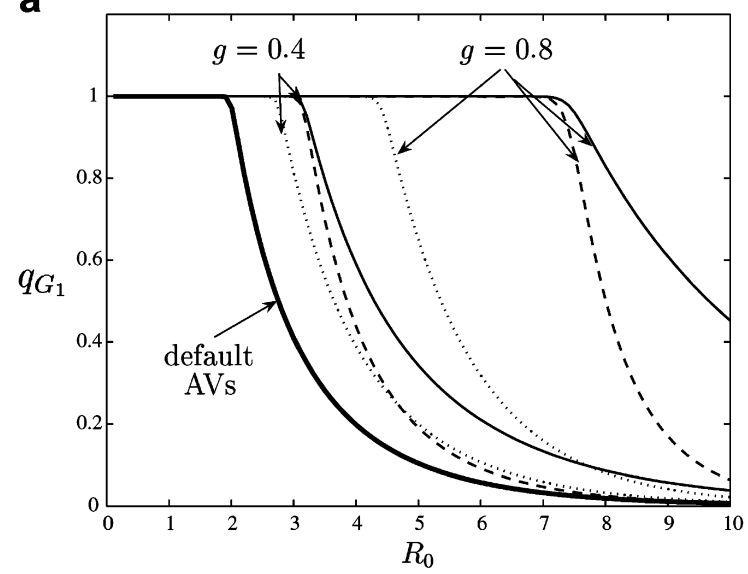

b

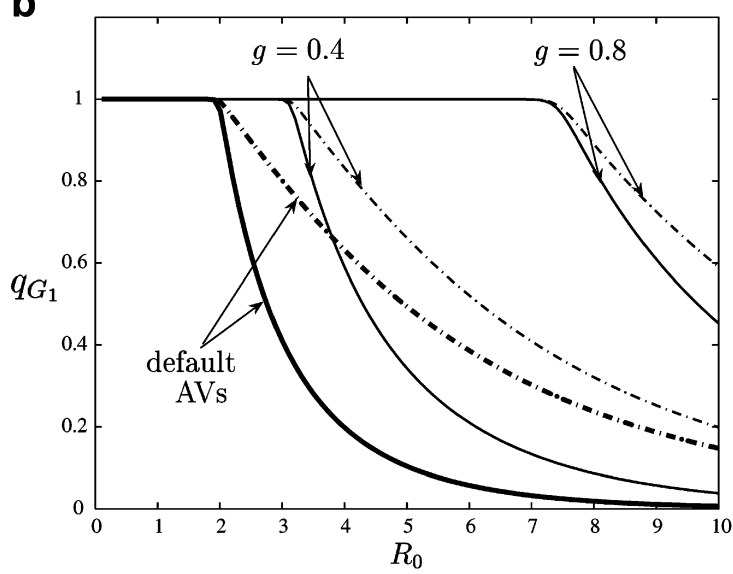

Fig. 2. (a) The effect of antiviral interventions on $q_{G_{1}}$ (probability of disease elimination initiated by a type $G_{1}$ infective) when the assumed offspring distribution is Poisson. Heavy solid curve: 'default AVs' strategy; light solid curves: Strategy A (pre-exposure distribution of AVs); dashed curves: Strategy B (delayed pre-exposure distribution of AVs); dotted curves: Strategy C (post-exposure distribution of AVs). (b) The effect of Strategy A on $q_{G_{1}}$ for two offspring distributions. Solid curves: assumed Poisson offspring distribution; dash-dotted curves: assumed Negative Binomial offspring distribution with the same mean as the Poisson distribution, but a variance 3 times that of the associated Poisson process without intervention. (Parameters as in Table 1.)

distribution allows a quantification of the impact of greater heterogeneity and the influence of super-spreaders within the underlying population, through the effect of an increased variance.

Fig. 2(b) compares, for Strategy A, the results between the two assumed offspring distributions and illustrates a Poisson offspring distribution as the limiting case, predicting the lowest values for $q_{G_{1}}$. With the mean fixed, increases in variance result in an increase in $q_{G_{1}}$, as was observed by [22], although $R$ is independent of the choice of distribution. The implications are that, for $R>1$, heterogeneity and the occurrence of super-spreaders (and hence greater variance) in the underlying population, lead to a decrease in the likelihood of a major outbreak from a single importation.

These results, concerning the impact on $R$ and the probability of a minor outbreak of postexposure antiviral distribution, agree with the findings from the simulation studies of [5] and [4]. From above and [5], efficient diagnosis, and rapid response, are critical to maximise the effectiveness of intervention strategies.

\section{The role of health care workers in transmission}

We consider the impact of protection measures for influenza-dedicated health care workers (the 'default AVs' strategy) on disease transmission and their role in early case numbers. Such results have consequences for pandemic planning decisions concerning not only the protection of workers to maintain a stable health care system, but also the protection of their families and the likelihood of a major outbreak.

In matrix $M$ we set $g=0$ thereby considering 3 classes of individuals. From Fig. 3(a), it is clear that if individuals are isolated after they have experienced half their infectivity $(f>0.5)$, there is 

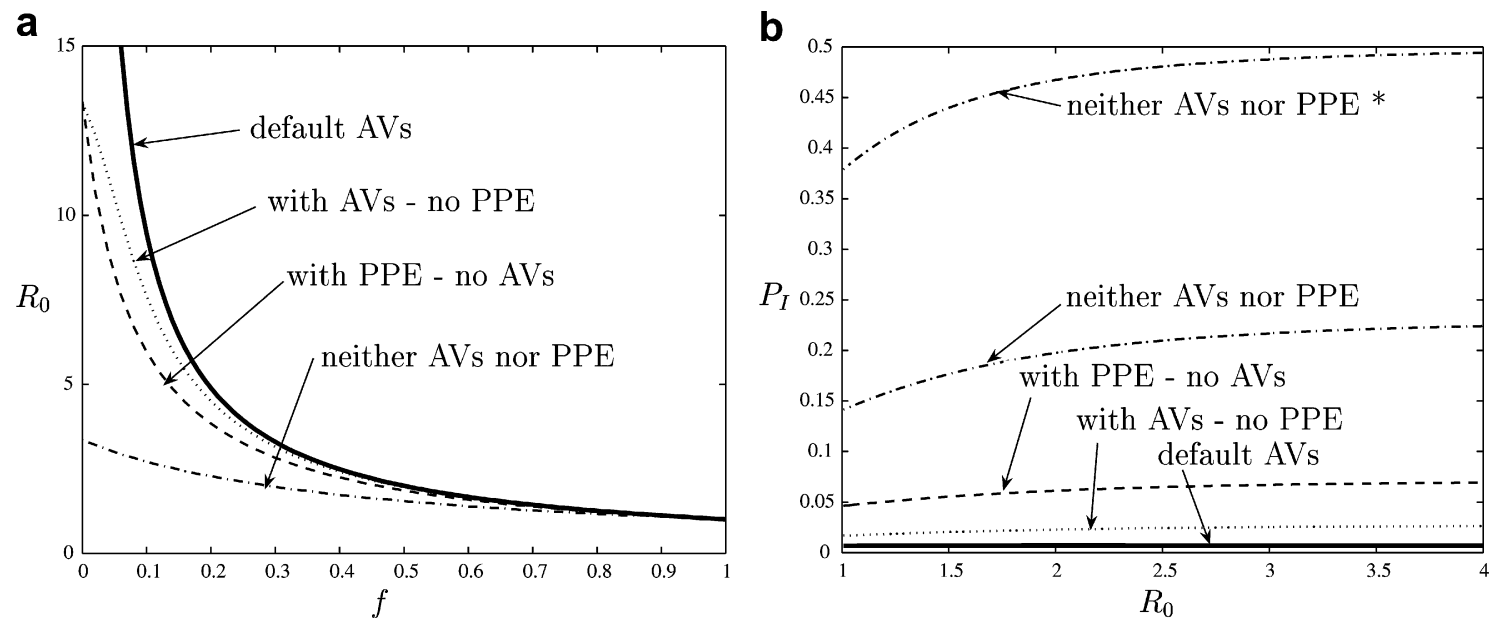

Fig. 3. The effect of interventions to protect health care workers. (a) Contours for $R=1$ comparing interventions: above the curves there is a chance that the disease takes off, while below, the outbreak is minor with certainty. Solid curve: 'default AVs' strategy; dotted curve: antiviral prophylaxis for health workers without PPE; dashed curve: PPE for health workers without antiviral distribution; dash-dotted curve: no intervention for health workers. In all cases diagnosed patients are treated with antiviral drugs. (b) Given initial infective proportions from each class $\left(G_{0}=0.988\right.$, $D_{0}=0.01$ and $H_{0}=0.002$ ), the mean proportion of infections directly attributable to a doctor or health care worker $P_{I}$, within the first four generations of an epidemic, is plotted for $f=0.5$. Line-styles and interventions are as in (a), and diagnosed patients are treated with antivirals in all cases except that labelled *. (Parameters as in Table 1 with $g=0$.)

little impact on overall disease transmission by the protection measures for health care workers; only for small values of $f$, say $f<0.3$, are the gains notable.

Although $R$ may not be significantly reduced through protective intervention for health workers, $P_{I}$ (the mean proportion of infections in the early stages of an epidemic that can be directly attributable to these classes) is disproportionately large (Fig. 3(b)). $P_{I}$, in the case of no intervention, exceeds by an order of magnitude the combined proportion of the health care classes within the population of approximately $1.2 \%$ (see Table 1). The 'default AVs' strategy redresses this disparity. We note further that not treating diagnosed patients on isolation (labelled *), more than doubles $P_{I}$ when health care workers are not protected. The implications are that health care workers, and as a consequence their families, are at far greater risk of infection than the general population. Implementing the 'default AVs' strategy protects health workers, their families and contacts, reducing $P_{I}$ below 0.012 (their proportional representation in the population) for $f=0.5$.

The significant role of health care workers in early transmission suggests that protecting health workers can reduce the probability of a minor outbreak $(q)$ appreciably. For example, in the case $f=0.5$ and $R_{0}=2$, the 'default AVs' strategy increased $q$ from 0.6 to 1 . For $f \geqslant 0.8$, the impact was marginal.

These results suggest that the 'default AVs' strategy is critical, not only to maintain a stable health care system, but to protect the families of health workers who would otherwise be at an order of magnitude greater risk than others in the population. 


\section{The role of children in transmission}

Children are commonly considered to exhibit closer mixing with other children than with, or between, adults, and thus we consider the potential impact of distributing antiviral drugs to, or vaccinating, this group. As mentioned, our choice of the range for the mixing rate $(\alpha)$ among children is guided by the relative rates of infection observed among children and adults in the two most recent influenza pandemics.

In matrix $M$ let $g=g_{c}=0.18$ denote the proportion of children within the community (Table 1 ), and consider the impact on transmission of supplying antivirals to all children for prophylaxis (Fig. 4(a)). By choosing the mixing rate $\alpha$ between 1.9 and 5.4, we consider a scenario bounded by the relative attack rates observed in the pandemics of 1968/9 and 1957, respectively [19]. The impact of this strategy is a modest reduction in $R$. Likewise, we observed a modest impact of this strategy on the proportion of early infectives attributable to children and on the probability of a minor outbreak $(q)$, for $1.9 \leqslant \alpha \leqslant 5.4$.

These results indicate that providing antivirals to children is not an effective strategy for transmission control, because the modest reduction in $R$ depicted in Fig. 4(a) requires antivirals to be administered to all children (18\% of the population) continuously. This drain on the limited antiviral stockpile is likely to be an unacceptable. However, if the disease risk in children is much higher than in adults, then a strategy that targets children needs to be considered.

Compared with using antivirals, administering a perfect vaccine to all children provides a value of $R$ that is only minimally smaller (Fig. 4(b)). In practice vaccines are not fully protective [23,24], but this extreme assumption provides a useful upper bound on the size of the effect. Compared

a

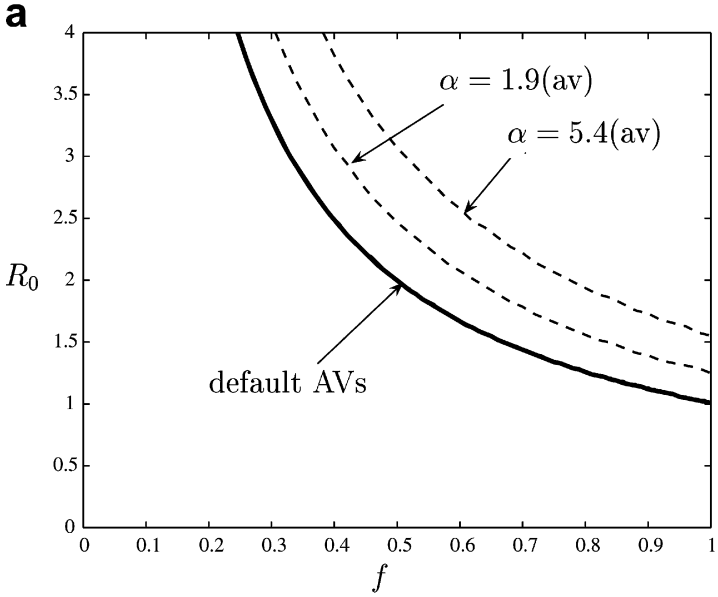

b

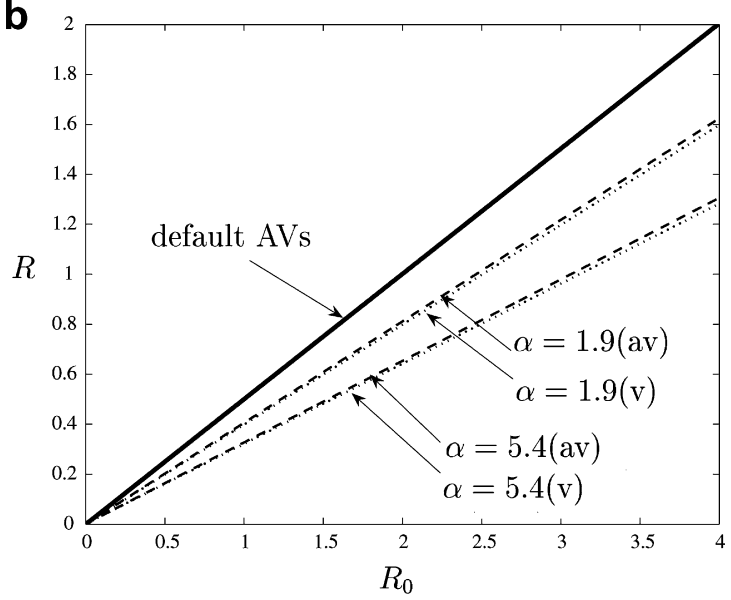

Fig. 4. The effect of interventions to protect children. (a) Contours for $R=1$ comparing interventions: above the curves there is a chance that the disease takes off, while below, the outbreak is minor with certainty. Solid curve: 'default AVs' strategy; dashed curves: Antivirals distributed as prophylaxis to children. (b) The impact on $R$ of strategies targeting children. Solid curve: 'default AVs' strategy; dashed curve: antivirals distributed as prophylaxis to children; dotted curve: children given vaccination. All illustrated strategies include the 'default AVs' case. Parameters as in Table 1 with $\left(\tilde{e}_{i}, \tilde{e}_{s}\right)=\left(e_{i}, e_{s}\right)$ for antiviral distribution $(\mathrm{av})$ and $(0,0)$ for the vaccine $(\mathrm{v})$. 
with the 'default AVs' strategy, vaccinating children reduces $R$ by approximately $20 \%$ when $\alpha=1.9$, and approximately $35 \%$ when $\alpha=5.4$ (see Appendix A).

These results contrast with those of [4] who predict that the impact of vaccinating $80 \%$ of children is comparable to vaccinating $80 \%$ of the general population. Longini et al. [4] use a more elaborate model, but also calibrated their parameters against the relative attack rates in the 1957 and 1968/9 pandemics. The apparent discrepancy in these conclusions seems to be due to the fact that their simulations are restricted to basic reproduction numbers less than 2 , for which each of these strategies drives $R$ below unity (see Fig. 4(b)). Our conclusions are stated in terms of reduction in $R$ over a wider range of values. For values of $R<2$ we get comparable eventual attack rates.

\section{Conclusions}

Estimates of $R_{0}$ for pandemic influenza strains, based on eventual attack rates [19] and growth rates for the epidemic $[7,4]$ in different locations, suggests a value of about 2 . Our results indicate that using antiviral drugs for targeted prophylaxis can contain an influenza strain with such a value of $R_{0}$, when we assume that the effectiveness of antivirals for the emerged influenza strain is the same as has been estimated for seasonal influenza. This relies on timely distribution of the antivirals to identified contacts of diagnosed cases.

As might be expected, under the assumption that cases are cared for by influenza-dedicated health care workers following diagnosis, our results suggest that health care workers contribute disproportionately to the transmission of the infection when they are not protected against infection. Our calculations provide reassurance that protecting health workers by treating cases with antiviral drugs, providing health workers with antivirals for pre-exposure prophylaxis and providing them with personal protective equipment greatly reduces the risk to health care workers. This in turn protects health care workers' families and is likely to enable the health care system to continue to function.

It is often proposed that control strategies that target children should be implemented. However, we found that the effectiveness of such strategies is only modest. This is explained by two facts, namely that

1. for pandemic influenza all people tend to be susceptible [25], in contrast to endemic infections that are transmitted primarily by children because infection provides lasting immunity, and

2. the observed attack rates in children, relative to adults, in past pandemics are not sufficiently extreme to suggest that children are responsible for a much greater fraction of the transmission [19].

However, while control strategies that focus on the protection of children have only a modest impact on the overall transmission, they clearly reduce the incidence among children. Therefore, protecting children from infection becomes a good strategy if disease in children is more severe.

The consequences of our results on $q$, the probability that an imported infection is contained, are limited. Firstly, if interventions are adequate for elimination, then this is indicated by $R$ being 
below unity. Secondly, if $R>1$ and importations of the infection occur every few days then it is inevitable that one of them leads to a major local epidemic. The results on $q$ therefore have practical consequences only for isolated importations, during the early stages of a pandemic. However, our results do indicate that providing antiviral drugs in the early stages can decrease the probability of an outbreak considerably, particularly if antivirals are distributed to contacts pre-exposure (Strategy A). Further, with importation, it is plausible that this strategy could delay an outbreak.

It is of interest, as has been observed by [22], that the concern about super-spreaders per se is misplaced in the sense that given an $R_{0}, q$ is generally larger the greater the heterogeneity among infectives in their potential to transmit the infection.

We note that, in the results reported above, we have not specifically dealt with asymptomatic cases. If they have limited infectivity then these results apply; however, if they are highly infectious then our model would need some adjustment.

Our approach has been to assess interventions on the basis of $R$ and $q$. One advantage of this approach is that calculations for a range of parameter values become more feasible than for microsimulation models.

\section{Appendix A. Estimating the leading eigenvalue of $M$}

For matrix (1), in the case protection is given to class $G_{2}$ so that $\tilde{e}_{i}=e_{i}$ and $\tilde{e}_{s}=e_{s}$, the leading eigenvalue $R$ is the maximum value of $\lambda$ that satisfies

$$
\begin{aligned}
0= & \lambda\left[\lambda^{3}-\lambda^{2}(\operatorname{Trace}(M))+\lambda\left(f^{2} m^{2} c(1-c)(\alpha-1)+f m^{2} e_{i} e_{s} e_{p}(1-c)(1-f)\left(1-e_{i}\right)\right.\right. \\
& \left.+f m^{2} c e_{i}^{2} e_{s}^{2} e_{p}(1-f)(\alpha-1)-p f m e_{i}(1-c)\right)-f^{2} m^{3} e_{i}^{2} e_{s}^{2} e_{p} c(1-c)(1-f)(\alpha-1)\left(1-e_{i}\right) \\
& \left.+p f^{2} m^{2} e_{i}^{2} e_{s}(1-c)(\alpha-1)-\left(1-(1-p)^{e_{i}}\right) f m c e_{i} e_{s}\right] .
\end{aligned}
$$

For parameter values as in Table 1 , the coefficients of $\lambda^{2}$ and $\lambda$ are negligible $\left(\mathrm{O}\left(10^{-3}\right)\right)$ when compared with the coefficients of $\lambda^{4}$ and $\lambda^{3}(\mathrm{O}(1))$. We can thus estimate $R$ with

$$
R \approx \operatorname{Trace}(M),
$$

whence $R$ is approximately linear in $m$.

In the case no protection is provided to class $G_{2}$ so that $\tilde{e}_{i}=1=\tilde{e}_{s}$, or this class is vaccinated so that $\tilde{e}_{i}=0=\tilde{e}_{s}$, results follow similarly that $R \approx \operatorname{Trace}(M)$.

\section{References}

[1] C. Ash, L. Roberts, Influenza: the state of our ignorance, Science 312 (2006) 379.

[2] D.S. Fedson, Pandemic influenza and the global vaccine supply, Clin. Infect. Dis. 36 (2003) 1552.

[3] N.G. Becker, K. Glass, Z. Li, G. Aldis, Controlling emerging infectious diseases like sars, Math. Biosci. 193 (2005) 205.

[4] I.M. Longini, E. Halloran, A. Nizam, Y. Pang, Containing pandemic influenza with antiviral agents, Am. J. Epidemiol. 159 (7) (2004) 623. 
[5] N.M. Ferguson, D.A.T. Cummings, S. Cauchemez, C. Fraser, S. Riley, A. Meeyai, S. Lamsirithaworn, D.S. Burke, Srategies for containing an emerging influenza pandemic in southeast Asia, Nature 437 (2005) 209.

[6] J.T. Wu, S. Riley, C. Fraser, G.M. Leung, Reducing the impact of the next influenza pandemic using householdbased public health interventions, PLoS Med. 3 (9) (2006).

[7] T.C. Germann, K. Kadau, I.M. Longini, C.A. Macken, Mitigation strategies for pandemic influenza in the United States, PNAS 103 (15) (2006) 5935.

[8] M. Nuño, G. Chowell, A.B. Gumel, Assessing the role of basic control measures, antivirals and vaccine in curtailing pandemic influenza: scenarios for the US, UK and the Netherlands, Journal of the Royal Society Interface, In press.

[9] O. Diekmann, J.A.P. Heesterbeek, Mathematical Epidemiology of Infectious Diseases: Model Building Analysis and Interpretation, Wiley, Chichester, 2000.

[10] G. Chowell, H. Nishiura, L.M.A. Bettencourt, Comparative estimation of the reproduction number for pandemic influenza from daily case notification data, J. R. Soc. Interface 4 (2007) 155.

[11] C.E. Mills, J.M. Robins, M. Lipsitch, Transmissibility of 1918 pandemic influenza, Nature 432 (2004) 904.

[12] I.M. Longini, A. Nizam, S. Xu, K. Ungchusak, W. Hanshaoworakul, D.A.T. Cummings, M.E. Halloran, Containing pandemic influenza at the source, Science 309 (2005) 1083.

[13] F.G. Hayden, J.J. Treanor, R.F. Betts, M. Lobo, J.D. Esinhart, E.K. Hussey, Safety and efficacy of the neuraminidase inhibitor GG167 in experimental human influenza, JAMA 275 (1996) 295.

[14] T. Jefferson, V. Demicheli, D. Rivetti, M. Jones, C. Di Pietrantoni, A. Rivetti, Antivirals for influenza in healthy adults: systematic review, Lancet 367 (2006) 303.

[15] F.G. Hayden, J.J. Treanor, R.S. Fritz, M. Lobo, R.F. Betts, M. Miller, N. Kinnersley, R.G. Mills, P. Ward, S.E. Straus, Use of the oral neuraminidase inhibitor oseltamivir in experimental human influenza, JAMA 282 (1999) 1240.

[16] R. Welliver, A.S. Monto, O. Carewicz, E. Schatteman, M. Hassman, J. Hedrick, H.C. Jackson, L. Huson, P. Ward, J.S. Oxford, Effectiveness of oseltamivir in preventing influenza in household contacts, JAMA 285 (2001) 748.

[17] M. Loeb, A. McGeer, B. Henry, M. Ofner, D. Rose, T. Hlywka, J. Levie, J. McQueen, S. Smith, L. Moss, A. Smith, K. Green, S.D. Walter, SARS among critical care nurses, Toronto, Emerg. Infect. Dis. 10 (2004) 251.

[18] M.-Y. Yen, Y.-E. Lin, I.-J. Su, F.-Y. Huang, et al., Using an integrated infection control strategy during outbreak control to minimize nosocomial infection of severe acute respiratory syndrome among healthcare workers, J. Hosp. Infect. 62 (2006) 195.

[19] L.E. Davis, G.G. Caldwell, R.E. Lynch, R.E. Bailey, T.D.Y. Chin, Hong Kong influenza: the epidemiologic features of a high school family study analyzed and compared with a similar study during the 1957 Asian influenza pandemic, Am. J. Epidemiol. 92 (1970) 240.

[20] K.G. Nicholson, R.G. Webster, A.J. Hay, Textbook of Influenza, 2nd ed., Blackwell, Oxford, 2000.

[21] W.J. Edmunds, C.J. O'Callaghan, D.J. Nokes, Who mixes with whom? A method to determine the contact patterns of adults that may lead to the spread of airborne infections, Proc.: Biol. Sci. 264 (1384) (1997) 949.

[22] J.O. Lloyd-Smith, S.J. Schreiber, P.E. Kopp, W.M. Getz, Superspreading and the effect of individual variation on disease emergence, Nature 438 (2005) 355.

[23] T. Jefferson, D. Rivetti, A. Rivetti, M. Rudin, C. Di Pietrantoni, V. Demicheli, Efficacy and effectiveness of influenza vaccines in elderly people: a systematic review, Lancet 366 (2005) 1165.

[24] R.E. Thomas, T.O. Jefferson, V. Demicheli, D. Rivetti, Influenza vaccination for health-care workers who work with elderly people in institutions: a systematic review, Lancet Infect. Dis. 6 (2006) 273.

[25] J.S. Nguyen-Van-Tam, A.W. Hampson, The epidemiology and clinical impact of pandemic influenza, Vaccine 21 (2003) 1762. 